\title{
Variations in Lateral Sphenoid Sinus Wall Defects
}

\author{
Gabriel Nakache $^{1}$ Arkadi Yakirevitch $^{1}$ Lev Bedrin $^{1}$ \\ ${ }^{1}$ Department of Otolaryngology, Head and Neck Surgery, The Sheba \\ Medical Center, Tel-Hashomer, Israel \\ Address for correspondence Gabriel Nakache, MD, Department of \\ Otolaryngology, Head and Neck Surgery, The Sheba Medical Center, \\ Indian J Neurosurg 2015;4:98-101. \\ Tel-Hashomer, Israel (e-mail: nakachega@gmail.com).
}

\begin{abstract}
Objective The article aims to present different forms of lateral sphenoid sinus wall defects.

Study Design Case series and literature review.

Methods A comparison between two patients who presented with spontaneous CSF rhinorrhea, defects in the lateral wall of the sphenoid sinus, and meningeal contents in the sphenoid sinus based on MRI is discussed. Both patients were operated endoscopically, through a trans-nasal-ethmoid-pterygoid approach.

Results In the first patient, a meningoencephalocele, protruding through a defect in the lateral sphenoid sinus wall and pterygoid base, occupied the inferior part of the sphenoid sinus. In the second patient, there were no exposed meningeal contents inside the sphenoid sinus. Instead, the lateral sphenoid sinus wall was thin and bulging medially into the inferior part of the sinus, with the dura and temporal lobe covered with thin bony cap.

Keywords

- sphenoid sinus

- lateral wall defect

- meningoencephalocele

Conclusion Complete dehiscence of the lateral wall of the sphenoid sinus results in exposed meningeal contents inside the sphenoid sinus. In partial dehiscence, the thin and weakened lateral wall of the sphenoid sinus yields to the pressure of the temporal lobe and bulges into the sphenoid sinus cavity. There are no other descriptions of partially dehiscent lateral sphenoid sinus walls in the literature.
\end{abstract}

\section{Introduction}

Defects in the lateral wall of the sphenoid sinus are a rare cause of cerebrospinal fluid (CSF) rhinorrhea. The etiology of these defects may be congenital or acquired. A dehiscent lateral craniopharyngeal canal, or Sternberg canal, arises from a malunion of two parts of the developing sphenoid bone: the greater wing and the basisphenoid. ${ }^{1,2}$ Originally reported in $4 \%$ of healthy adults, ${ }^{3}$ its true prevalence is not known, as is its relation with adult-onset CSF rhinorrhea and meningoceles of the sphenoid sinus. Acquired conditions probably account for the larger part of lateral sphenoid sinus wall defects, and include trauma, adjacent tumor, idiopathic intracranial hypertension, and iatrogenic damage.

received

April 13, 2015

accepted

May 14, 2015

published online

July 28, 2015
Regardless of their cause, large defects in the lateral sphenoid sinus wall are often accompanied by a temporal lobe meningocele or meningoencephalocele. When the defect is small, only CSF would leak, without dural herniation. ${ }^{4}$ In both cases, the defect should be repaired, achieving high rates of success with both transcranial and endoscopic approaches. ${ }^{5-9}$

Ongoing pressure plays a role in the pathogenesis of lateral sphenoid sinus wall defects. We hypothesize that apart from complete dehiscence, cases of weakened partially dehiscent walls, bulging into the sphenoid sinus cavity, with micro-tears allowing for CSF leak but without exposed meningeal contents, should also exist. This entity has not been described in the literature so far.
License terms of India 


\section{Methods}

A comparison of two patients presented with idiopathic CSF rhinorrhea confirmed by positive $\beta_{2}$-transferrin tests is discussed. In both patients, computed tomography (CT) showed an apparent defect of the lateral sphenoid sinus wall, with no underlying extensive pneumatization of the lateral recess. The magnetic resonance imaging (MRI) demonstrated intracranial contents in the sphenoid sinus cavity. Both patients underwent endoscopic surgery, with a trans-nasal-ethmoid-pterygoid approach to the lateral recess of the sphenoid sinus, and multilayer reconstruction of the bony defect. Both patients had continuous lumbar drainage intra- and postoperatively.

\section{Results}

\section{Patient 1}

A 57-year-old woman, overweight but otherwise healthy, presented with a 6-month history of spontaneous clear rhinorrhea, with no previous head trauma or surgery. During that period she suffered from two episodes of pneumococcal meningitis, which were successfully treated with intravenous (IV) ceftriaxone each time. CSF leakage was confirmed by a positive $\beta_{2}$-transferrin test. A high-resolution CT demonstrated total opacification of both sphenoid sinuses, a $6-\mathrm{mm}$ defect in the lateral wall of the left sphenoid sinus, and a 9-mm defect in the roof of the pterygoid base (-Fig. 1). T2-weighted MRI images showed a temporal lobe meningoencephalocele occupying the lower half of the left sphenoid sinus and the base of the pterygoid plates (-Fig. 2). An endoscopic trans-nasal-ethmoidpterygoid approach was chosen to open the entire left sphenoid sinus. After removal of the sphenoidal rostrum and dissection of the pterygopalatine fossa, the meningoencephalocele was exposed (-Fig. 3). The latter was shrunk by electrocautery and resected at its base, and the bony defect was sealed by a multilayer reconstruction using an abdominal fat plug and an overlying free septal mucosal graft.

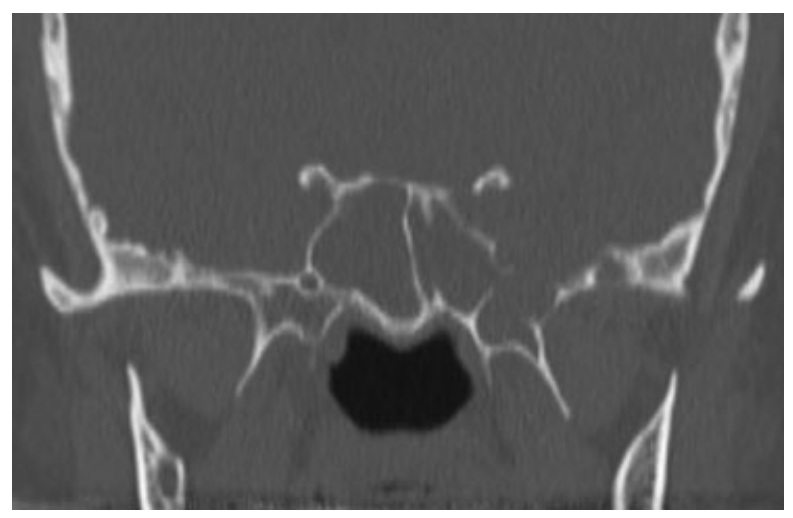

Fig. 1 High-resolution CT image of patient 1, showing a defect in the lateral wall of the left sphenoid sinus and an accompanying defect in the base of the pterygoid plates. Both sphenoid sinuses are totally opacified.

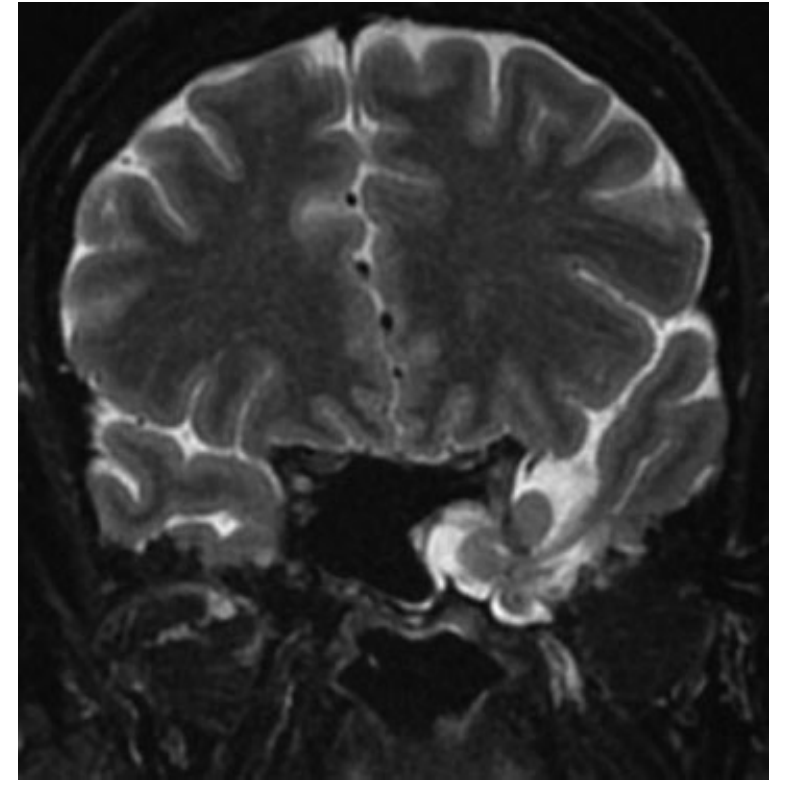

Fig. 2 Coronal T2-weighted MRI image of patient 1, demonstrating a temporal lobe meningoencephalocele occupying the inferior part of the left sphenoid sinus and the left pterygoid base. The right sphenoid sinus is well aerated.

\section{Patient 2}

A 43-year-old otherwise healthy man presented with an uncomplicated 3-week-old episode of spontaneous left rhinorrhea approved as CSF leak by means of $\beta_{2^{-}}$ transferrin test. There was no previous history of head trauma or surgery. His high-resolution CT scan revealed a $10-\mathrm{mm}$ defect in the inferior part of the lateral wall of the left sphenoid sinus and a bony septum traversing the sinus cavity. The left sphenoid sinus was totally opacified, whereas the right sphenoid sinus was partially obstructed. The

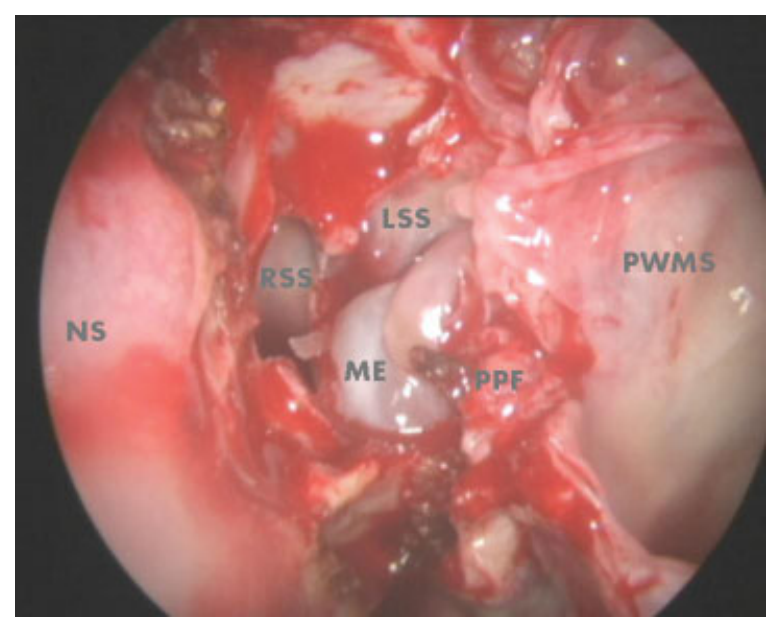

Fig. 3 Patient 1. Endoscopic view of the meningoencephalocele (ME), after resection of the middle concha, posterior septum, sphenoidal rostrum, medial wall of maxillary sinus, and sphenopalatine artery. Note the pterygopalatine fossa (PPF), posterior wall of the maxillary sinus (PWMS), nasal septum (NS), and right and left sphenoid sinuses (RSS, LSS). 


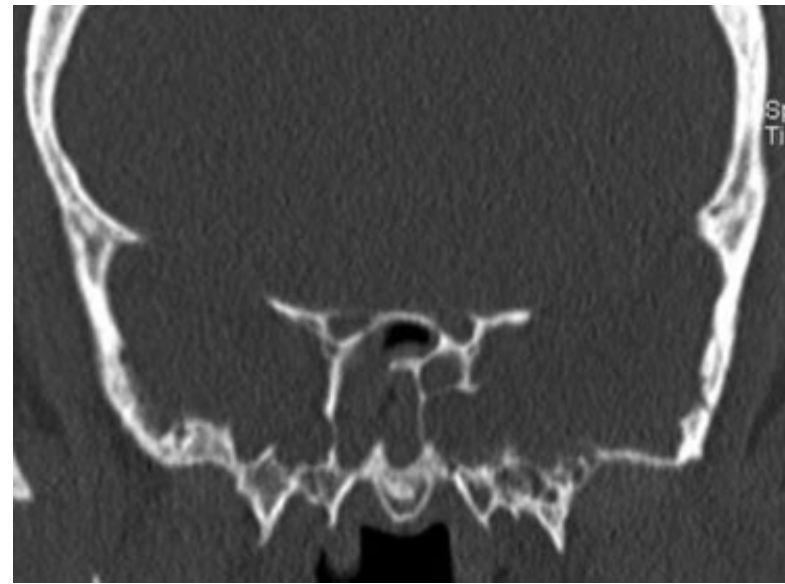

Fig. 4 High-resolution CT image of patient 2. Note the large defect in the left lateral sphenoid sinus wall, the transverse bony septum inside the left sphenoid sinus cavity, and the intact pterygoid plates bilaterally. The left sphenoid sinus is totally opacified. The right sphenoid sinus is partially aerated.

pterygoid plates were intact bilaterally (-Fig. 4). MRI demonstrated meningeal contents in the left sphenoid sinus (-Fig. 5). Similar to the first patient, a continuous lumbar drainage was installed, the left sphenoid sinus was reached by an endoscopic trans-nasal-ethmoid-pterygoid approach. Unlike the first patient, after the entire left sphenoid sinus cavity was revealed, we found no exposed intracranial contents, but rather a bony bulge occupying the inferolateral part of the sinus. No frank CSF leak was evident. Carefully breaching that thin layer of bone, we revealed the middle fossa dura underneath (-Fig. 6). The entire bony shell was removed, and the meningocele shrunk by electrocautery and resected at its base. The 1-cm large lateral wall defect was then bridged in a multilayer fashion, using septal cartilage and a contralateral nasoseptal vascularized flap.

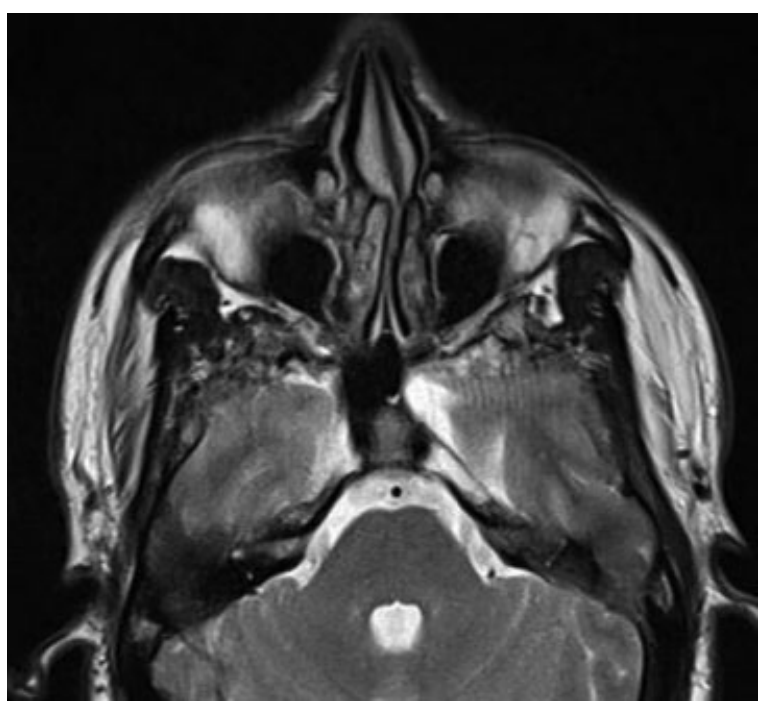

Fig. 5 Axial T2-weighted MRI image of patient 2. Meningeal contents bulge into the left sphenoid sinus cavity.

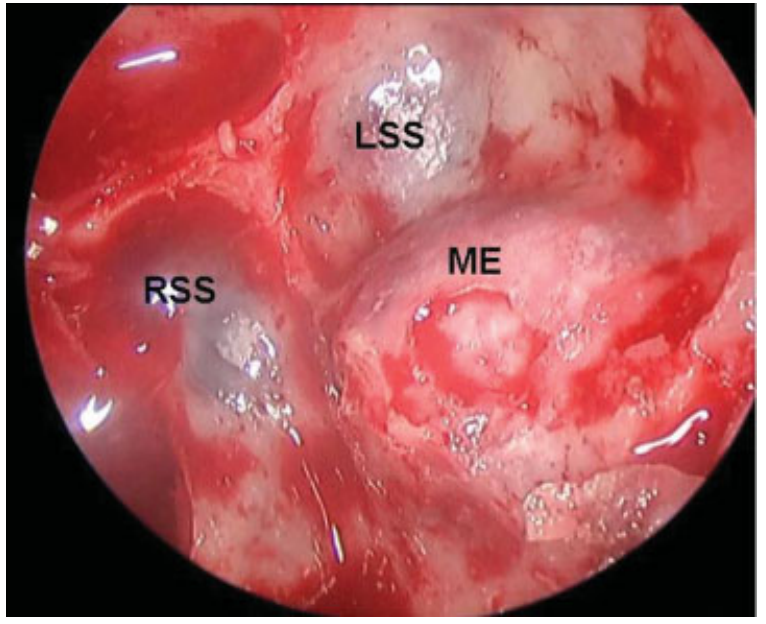

Fig. 6 Right and left sphenoid sinuses (RSS, LSS) of patient 2. The inferior part of the left sphenoid sinus is occupied by a meningoencephalocele (ME), covered by a thin layer of bone (here punctured by a suction catheter to expose the dura).

\section{Discussion}

The pathogenesis of defects in the lateral wall of the sphenoid sinus is complex. In some cases, such as trauma or surgery, the bony wall is disrupted by an abrupt insult. In other cases involving elevated intracranial pressure, progressive pressure gradually weakens the bone, ultimately creating a complete defect. In pure congenital cases, a developmental error in the ossification of different parts of the sphenoid bone results in a malformed lateral wall of the sphenoid sinus. Pathogenesis can be multifactorial, as proposed by Tabaee et al, when an asymptomatic congenital base of skull defect enlarges over time by continuous CSF pulsations, until the development of a dural tear, a meningoencephalocele, or both. ${ }^{5}$

Both transcranial and endoscopic approaches were described to seal the CSF leak and reconstruct the skull base defect. Evidence supports applicability of an endoscopic approach for most cases, reserving transcranial surgery for recurrent cases after failed endoscopic attempts. ${ }^{2,5-8}$

The rising number of reports in recent years suggests that lateral sphenoid sinus wall defects are perhaps not as rare as previously thought. ${ }^{5-7,9}$ In these reports, the sphenoid sinus is partially or entirely occupied by a meningoencephalocele, and the lateral wall of the sphenoid sinus is absent.

Similar findings could be seen in our patient 1. - Fig. 3 shows the surgically exposed left sphenoid sinus occupied in its inferior part by a meningoencephalocele, originating from a fully dehiscent lateral sphenoid sinus wall.

Vaezi et al have described a different version of lateral sphenoid sinus wall defects, in which CSF collections or meningoceles were covered by normal sphenoid sinus mucosa. ${ }^{4}$ The lateral wall of the sphenoid sinus in these cases was either absent, accompanied by a meningocele, or intact with assumed micro-tears, with CSF collections.

Although two of the main pathogenetic pathways of lateral sphenoid sinus wall anomalies, congenital maldevelopment 
and gradual pressure, would result in a weak and thin lateral wall, descriptions of thin lateral walls bulging into the sphenoid sinus cavity are absent in the literature.

In our patient 2 , preoperative imaging supported the diagnosis of a lateral sphenoid sinus wall dehiscence with an accompanying meningoencephalocele in the sphenoid sinus. However, intraoperatively, we found a thin medially displaced lateral sphenoid sinus wall, without dural exposure (-Fig. 6). Because intracranial pressure (ICP) was lowered intraoperatively by a continuous lumbar drain, there was no evident CSF leak in the operative field. We believe that although there was no apparent dehiscence of the skull base, CSF leaked to the nose through microscopic defects in the thin medially bulging lateral wall. Removing this bony cap and the dural contents behind it confirmed the absence of a normal lateral sphenoid sinus wall. When we reviewed the patient's preoperative CT scan, the medially displaced lateral sphenoid sinus wall was visible as the transverse septa crossing the sinus cavity (-Fig. 4).

\section{Conclusion}

When evaluating a patient with an apparent meningoencephalocele in the sphenoid sinus, surgeons should take into consideration the possibility of encountering a partially, rather than completely, dehiscent lateral sphenoid sinus wall.

\section{References}

1 Schick B, Brors D, Prescher A. Sternberg's canal-cause of congenital sphenoidal meningocele. Eur Arch Otorhinolaryngol 2000;257(8):430-432

2 Catala M. Développement embryonnaire du sphénoïde [in English]. J Neuroradiol 2003;30(4):196-200

3 Lai SY, Kennedy DW, Bolger WE. Sphenoid encephaloceles: disease management and identification of lesions within the lateral recess of the sphenoid sinus. Laryngoscope 2002;112(10): 1800-1805

4 Vaezi A, Snyderman CH, Saleh HA, Carrau RL, Zanation A, Gardner P. Pseudomeningoceles of the sphenoid sinus masquerading as sinus pathology. Laryngoscope 2011;121(12):2507-2513

5 Tabaee A, Anand VK, Cappabianca P, Stamm A, Esposito F, Schwartz TH. Endoscopic management of spontaneous meningoencephalocele of the lateral sphenoid sinus. J Neurosurg 2010;112(5):1070-1077

6 Castelnuovo P, Dallan I, Pistochini A, Battaglia P, Locatelli D, Bignami M. Endonasal endoscopic repair of Sternberg's canal cerebrospinal fluid leaks. Laryngoscope 2007;117(2):345-349

7 Al-Nashar IS, Carrau RL, Herrera A, Snyderman CH. Endoscopic transnasal transpterygopalatine fossa approach to the lateral recess of the sphenoid sinus. Laryngoscope 2004;114(3): 528-532

8 Bendersky DC, Landriel FA, Ajler PM, Hem SM, Carrizo AG. Sternberg's canal as a cause of encephalocele within the lateral recess of the sphenoid sinus: a report of two cases. Surg Neurol Int 2011;2:171

9 Samadian M, Moghaddasi H, Vazirnezami M, et al. Transcranial approach for spontaneous CSF rhinorrhea due to Sternberg's canal intrasphenoidal meningoencephalocele: case report and review of the literature. Turk Neurosurg 2012;22(2):242-245 